\title{
Origin and Structure of the Quasi-Landau Resonances
}

\author{
Jarbas C. Castro, Myron L. Zimmerman, Randall G. Hulet, and Daniel Kleppner \\ Research Laboratory of Electronics and Department of Physics, Massachusetts Institute of Technology, \\ Cambridge, Massachusetts 02139 \\ and \\ Richard R. Freeman \\ Bell Telephone Laboratories, Holmdel, New Jersey 07733 \\ (Received 18 August 1980)
}

\begin{abstract}
Highly resolved spectra are presented which show how the low-magnetic-field energylevel structure of a single-electron atom gives rise to the quasi-Landau resonances as the field is increased. Data from the strong-mixing region where electric and magnetic forces are comparable reveal that evenly spaced energy levels are a general feature, irrespective of parity and magnetic quantum number of the states. The quasi-Landau resonances emerge by a concentration of oscillator strength into a single level of each term.
\end{abstract}

PACS numbers: $32.70 .-\mathrm{n}, 33.55 .+\mathrm{c}$

The absorption spectra of alkali and alkaline earth atoms in high magnetic fields can display a periodic structure extending above the ionization limit. The absorption lines, generally called the quasi-Landau resonances, were discovered by Garton and Tomkins ${ }^{1}$ and have been the subject of numerous theoretical ${ }^{2}$ and experimental ${ }^{3}$ studies. The major feature of the resonances, their characteristic energy spacing of $\frac{3}{2} \hbar \omega_{c}\left(\omega_{c}\right.$ is the cyclotron frequency) at the ionization limit, has been explained with a model in which the wave function is localized in a plane perpendicular to the magnetic field. ${ }^{4,5}$ With this approximation, the resonance structure emerges quite naturally from a WKB analysis. In spite of this success, however, the WKB approach suffers from a number of shortcomings. Although it can predict the position of the resonances, it cannot predict the detailed absorbtion spectrum which can be observed in high-resolution experiments nor is it suited for calculating oscillator strengths or lifetimes. Most seriously, it fails to shed light on the general question of the structure of atoms in strong magnetic fields.

By studying completely resolved spectra we have been able to observe experimentally how the low-field spectrum of Rydberg atoms evolves into the quasi-Landau spectrum as the field is increased, and we have been able to account quantitatively for our observations. We find that the quasi-Landau spectrum is formed from a subset of a general class of periodically spaced levels, heretofore unobserved. The particular levels which give rise to the quasi-Landau resonances are made conspicuous by a transfer of oscillator strength from the remaining levels, which sug- gests that the appearance of any particular resonance spectrum is governed by the optical excitation route employed. We believe, however, that the underlying system of periodically spaced levels characterizes the complete structure of the atom in the strong-mixing region where the electric and magnetic interactions are comparable.

Our experiment employs laser spectroscopy to study highly excited states of sodium in a magnetic field, using a procedure previously described. ${ }^{6}$ The atoms are observed in an atomic beam directed along the axis of a magnet. The axial atomic-beam geometry reduces the motional electric field to less than $0.5 \mathrm{~V} / \mathrm{cm}$, and essentially eliminates the complexities of the motional Stark effect. The atoms are excited in a two-step process by laser beams directed perpendicular to the magnetic field. The value of $m_{l}$ in the final state is determined by the polarization of the laser beams. The highly excited atoms are detected by ionizing them with a pulsed electric field and detecting the electrons.

The Hamiltonian of an alkali atom in a magnetic field is

$$
H=\frac{1}{2} p^{2}-1 / r+\frac{1}{2} \alpha m_{l} B+\frac{1}{8} \alpha^{2} B^{2}\left(x^{2}+y^{2}\right),
$$

where we have suppressed spin-dependent terms and perturbations to the potential at small $r$ because of the core electrons. The diamagnetic term, $H_{d}=\frac{1}{8} \alpha^{2} B^{2}\left(x^{2}+y^{2}\right)$, has off-diagonal elements between Coulombic states of any principal quantum number $n$ and with $l$ differing by $0, \pm 2$. Consequently, the only "good" quantum numbers are parity and $m_{l}$. At low fields $H_{d}$ splits every term into a series of manifolds characterized by 


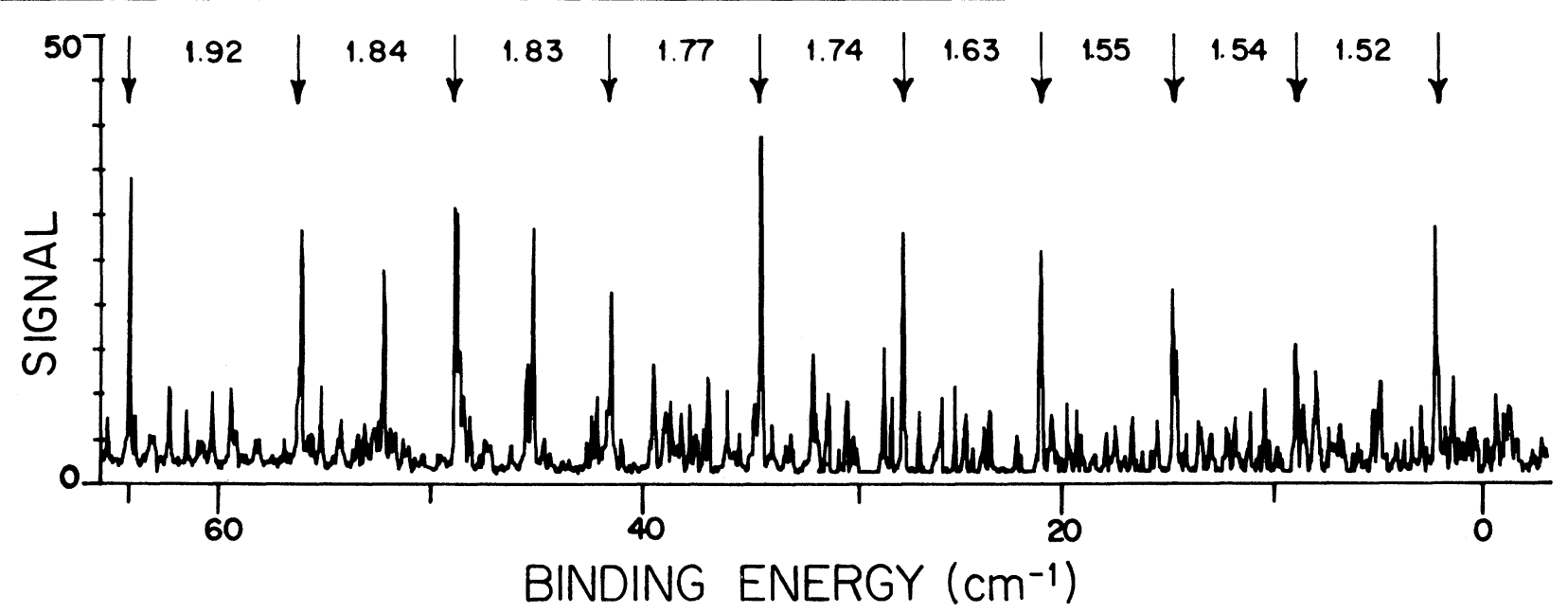

FIG. 1. Quasi-Landau spectrum observed by laser excitation and field ionization of even-parity, $m_{l}=-2$ states of sodium in a magnetic field of $4.2 \mathrm{~T}$. The arrows indicate the quasi-Landau levels. The intermediate peaks are due to other levels, as described in the text. The numbers between the arrows give the level separation in units of $\hbar \omega_{c}$. A WKB analysis predicts a spacing of 1.5 at $E=0$, increasing with binding energy in agreement with the data. Relative intensities are not reliable due to laser fluctuations.

$n, m_{l}$, and parity. In this region, the energy of each member of a manifold increases linearly with $B^{2}$, the slope depending on the particular eigenstate. At higher fields, $n$-mixing occurs and the energy dependence tends to become linear with $B$. At a field of $5 \mathrm{~T}_{,}\left\langle H_{d}\right\rangle$ is comparable with the Coulomb energy for $n=55$.

We have studied even-parity states with $m_{l}=-1$ and $m_{l}=-2$. The spectrum of a single laser scan at $4.2 \mathrm{~T}$ for the $m_{l}=-2$ states, Fig. 1 , clearly shows the quasi-Landau levels. (The selection rules allow transitions to $m_{l}=0$ states, but the oscillator strength is too weak for these transitions to appear in our data.) Figure 2 is a map of spectra for magnetic fields in the range 0 to $55 \mathrm{~T}$. The small linear Zeeman shift has been subtracted from the data. The intensity of the signal is proportional to the square of the $d$-state amplitude of the final state, since the second step of the optical excitation is $3 p \rightarrow n d$. At low field the $d$ character is concentrated among the higher levels of each manifold. As the field increases and the terms become mixed, the $d$ character transfers to the highest level of each manifold. The separation approaches $\frac{3}{2} \hbar \omega_{c}$ and the lines become completely resolved quasi-Landau resonances. It should be realized, however, that these highest levels are a subset of the complete manifold, and that their prominence is simply a consequence of the selection rule for the transition. The semiclassical method applies only to the highest level of each manifold.
Figure 3 is a map of spectra for even-parity states with $m_{l}=-1$. In contrast to the $m_{l}=-2$ states shown in Fig. 2 , the $d$-state character for these levels remains spread throughout the manifold as the field increases. Because every state is excited, the spectrum is so rich that it would look featureless under conditions of poor resolution or motional Stark mixing. There is a striking feature, however: The spectrum has the same periodicity as the $m_{l}=-2$ spectrum. The separation between corresponding levels of adjacent manifolds (i.e., highest, next lower, etc.) is the quasi-Landau spacing. Evidently, the simple periodic structure is a general property of all of the levels, not just those of states localized in the $x-y$ plane.

In the absence of any general theory for the problem of hydrogen in a magnetic field we have calculated the energies by diagonalizing the Hamiltonian using a zero-field spherical basis. Although such a technique is known to work well at low fields, ${ }^{6}$ it would seem poorly suited for the strong-mixing region. To our surprise, agreement between calculation and experiment is good, as Fig. 4 shows. The numerical method has been described previously, ${ }^{7}$ and further details will be published elsewhere. The unexpected accuracy of the approach gives hope that approximate solutions based on low-field and high-field basis can be matched in the strong-mixing region to provide a general solution.

The portion of this research carried out at the 


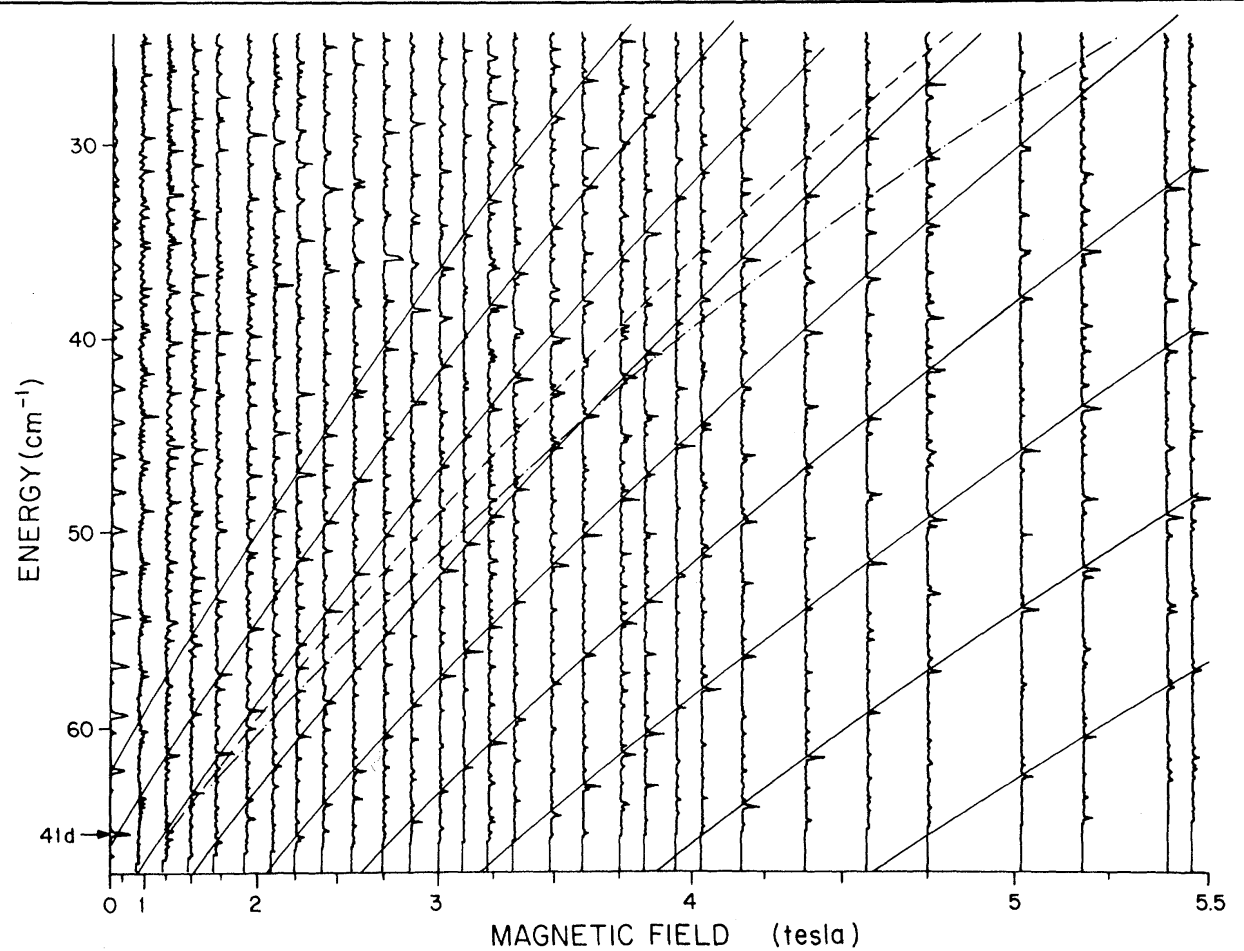

FIG. 2. Experimental excitation curves for even-parity, $m_{l}=-2$ energy levels of sodium plotted as a function of magnetic field (plotted on a squared scale). Signals generated by ionizing the excited atoms appear as horizontal peaks. The level structure is most clearly seen by sighting along the drawn lines with the eye close to the plane of the paper. Solid lines indicate the evolution of the highest level for several $n$ manifolds. The dashed and dot-dashed lines show the second- and third-highest levels of the $n=40$ manifold, respectively.

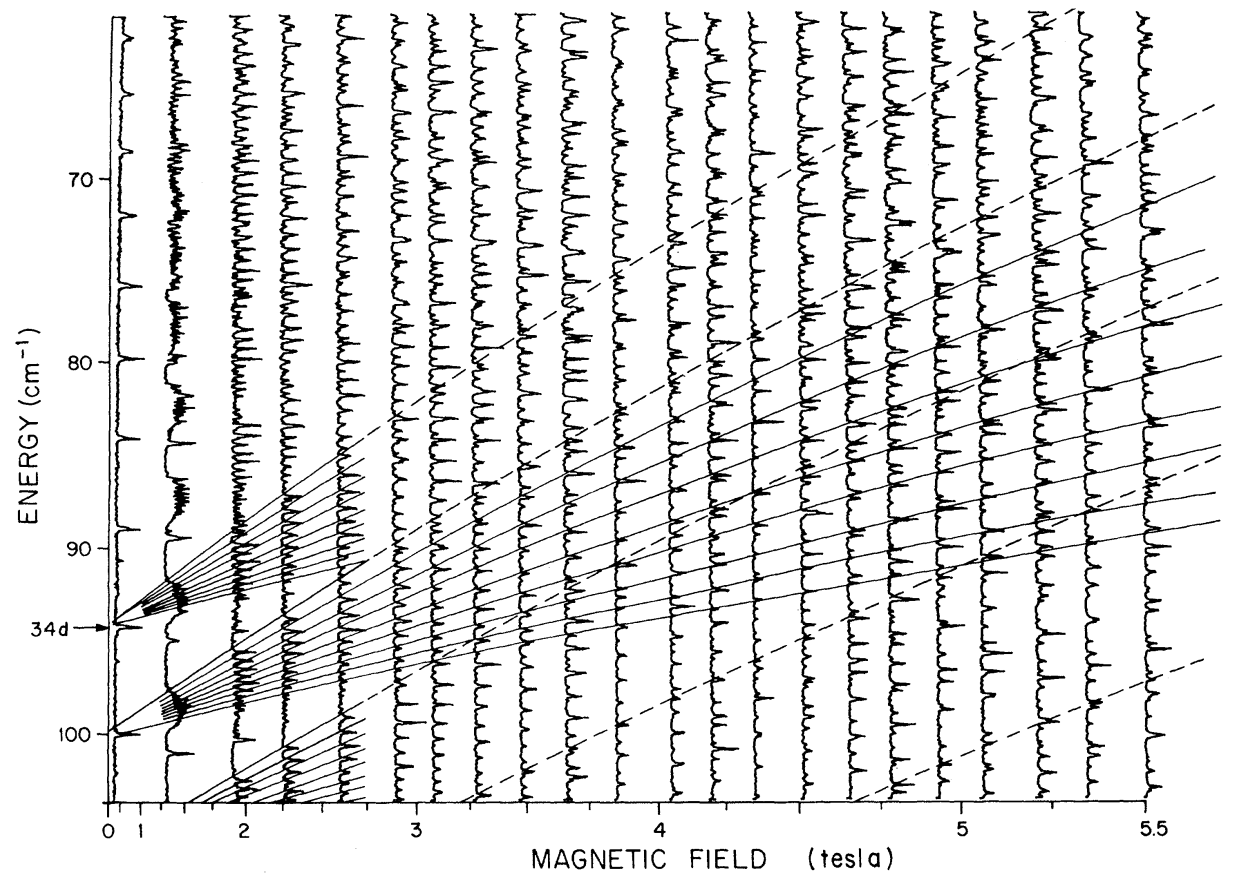

FIG. 3. Same as Fig. 2 except for even-parity, $m_{l}=-1$ energy levels and a different energy range. The dashed lines are drawn to indicate the evolution of the highest level for several $n$ manifolds. Note that the corresponding levels of adjacent $n$ manifolds are equally spaced. The level structure is most clearly seen by sighting along the drawn lines with the eye close to the plane of the paper. 


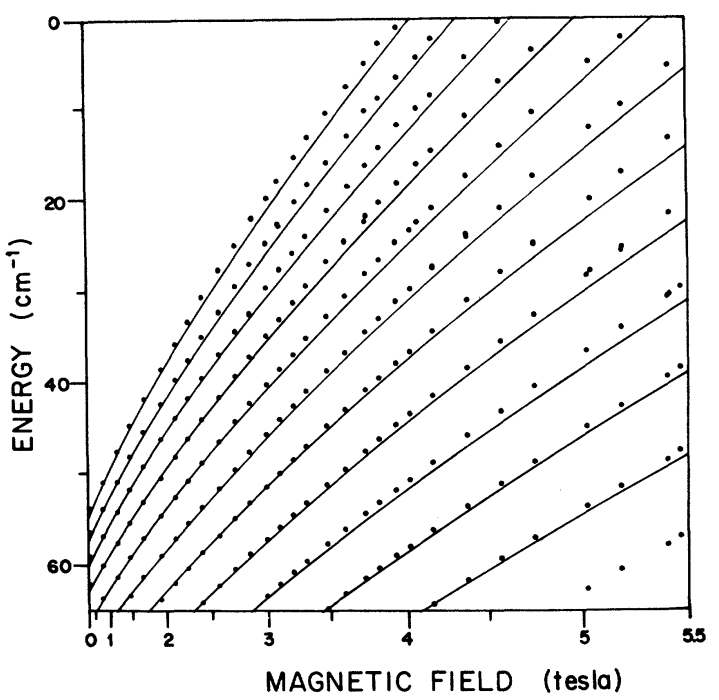

FIG. 4. A comparison of numerical results (solid lines) and experimental results (dots) for the highest level of each manifold for the even-parity, $m_{l}=-2$ states. The numerical results are obtained by a diagonalization using a spherical basis of Coulombic states, $n=25-75$ and $l=2,4$, and 6 . The systematic discrepancy, which is less than $1 \%$ of the diamagnetic energy, is largely due to the omission of higher- $l$ states.
Massachusetts Institute of Technology was sponsored by the National Science Foundation under Grant No. PHY79-09743. One of us (J.C.C.) thanks Conselho Nacional de Desenvolvimento Científico e Tecnológico (Brazil) for partial support.

${ }^{1}$ W. R. S. Garton and F. S. Tomkins, Astrophys. J . 158, 839 (1969).

${ }^{2}$ A. F. Starace and G. L. Webster, Phys. Rev. A $\underline{19}$, 1629 (1979), and references therein.

${ }^{3}$ K. T. Lu, F. S. Tomkins, and W. R. S. Garton, Proc. Roy. Soc. London, Ser. A 364, 421 (1978); R. J. Fonck et al., Phys. Rev. A 21, 861 (1980); N. P. Economou, R. R. Freeman, and P. F. Liao, Phys. Rev. A $\underline{18}, 2506$ (1978).

${ }^{4}$ A. R. Edmonds, J. Phys. (Paris), Colloq. 31, C4-71 (1970); A. R. P. Rau, Phys. Rev. A 16, 613 (1977).

${ }^{5}$ A. F. Starace, J. Phys. B 6,585 (1973).

${ }^{6}$ M. L. Zimmerman, J. C. Castro, and D. Kleppner, Phys. Rev. Lett. $\underline{40}, 1083$ (1978).

${ }^{7}$ M. L. Zimmerman, M. G. Littman, M. M. Kash, and D. Kleppner, Phys. Rev. A 20, 2251 (1979).

\title{
Experimental Potentials for the $X^{2} \Sigma^{+}$and $A^{2} \Pi$ States of $\mathrm{NaHe}$
}

\author{
M. D. Havey, (a) S. E. Frolking, and J. J. Wright \\ Physics Department, University of New Hampshire, Durham, New Hampshire 03821 \\ (Received 23 June 1980) \\ Experimental potentials for the $X^{2} \Sigma^{+}$and $A^{2} \Pi$ states of the NaHe molecule are presented. \\ The potentials are generated from the temperature dependence of the red wing of the $\mathrm{Na}$ \\ resonance line perturbed by He. For the $A^{2} \Pi$ state an unexpectedly large value is obtained \\ for $D_{e}=480(50) \mathrm{cm}^{-1}$ at $R_{e}=4.4(2) a_{0}$. The physical basis for this result, which indicates \\ an important role for the internal structure of the rare gas in determining the repulsive \\ part of the alkali-rare-gas interaction, is discussed.
}

PACS numbers: $34.20 .-\mathrm{b}$

In this Letter we report the results of our observations of the temperature dependence of the $A^{2} \Pi \rightarrow X^{2} \Sigma^{+}$emission spectrum of molecular NaHe. The spectrum, which is observed for $1100 \AA$ on the red side of the He-perturbed $\mathrm{Na}$ resonance doublet, is analyzed by using the quasistatic model, as developed by Gallagher and coworkers,${ }^{1-3}$ to yield potential curves for the two lowest states of NaHe. For the $A^{2} \Pi$ state we obtain a remarkably large value for $D_{e}$. A deep penetration of the $\mathrm{Na} 3 p \pi$ orbital by the $\mathrm{He}$ atom is responsible for this result, which suggests that the internal structure of the rare gas plays an important role in determining the repulsive part of the alkali-rare-gas interaction.

The interaction of alkali-rare-gas pairs has been studied extensively, with recent work focused on $\mathrm{NaNe}^{4-7}$ and $\mathrm{NaHe}^{8,9}$ The interaction potentials are relevant to interpretation of a variety of elastic and inelastic collision processes involving $\mathrm{Na}$ in the $3 p$ state. They are also important in evaluation of the suitability of the various alkali-rare-gas combinations for use in a possible rare-gas-metal vapor laser system. Reasonable accord has been reached between model potential calculations $\mathrm{s}^{6,7}$ and spectroscopic data $^{5,10}$ on the $X^{2} \Sigma^{+}$and $A^{2} \Pi$ states of NaNe. However, for the NaHe system calculations using 